\title{
Nitric Oxide-Assisted Atmospheric Pressure Corona Discharge Ionization for the Analysis of Automobile Hydrocarbon Emission Species
}

\author{
Mark A. Dearth and T. J. Korniski \\ Ford Motor Company, Dearborn, Michigan, USA
}

\begin{abstract}
Nitric oxide reagent gas has been found to improve the sensitivity and robustness of the atmospheric pressure corona discharge ionization (APCDI) process. Sensitivity has been increased by a factor of 20-100, depending on the compound, over APCDI without nitric nxide. The robustness (defined as the sensitivity to matrix interferences) of APCDI in the presence of water has been improved by a factor of 3 over normal APCDI. These improvements are due in part to a modification of the commercial inlet system and ionization chamber that allows the chamber and sample gases to be heated to 100 and $350{ }^{\circ} \mathrm{C}$. respectively. Nitric oxide was chosen as the reagent gas because of the variety and selectivity of its interaction with hydrocarbons with differing functional groups. Product ions of nitric oxide ionization and their subsequent tandem mass spectra are presented and discussed for selected alkanes, alkenes, alkylbenzenes, alcohols, aldehydes, and an ether. A tandem mass spectrometry (unique parent ion-daughter ion transition) method was developed to quantify compounds of specific interest in vehicle emissions. The absolute sensitivity for these compounds, under ideal conditions, was determined and ranges from $0.006 \mathrm{ppb}$ for xylene (most sensitive) to $80 \mathrm{ppb}$ for $\mathrm{C}_{8}$ (or larger) normal alkanes. Routine sensitivity for real-world samples was in the single parts per billion range for aromatic and olefinic species. Potential applications include the real-time, on-line monitoring of selected hydrocarbons in automobile exhaust. (J Am Soc Mass Spectrom 1994, 5, 1107-1114)
\end{abstract}

$\mathrm{M}$ easurement of individual aromatic, olefinic, and oxygenated hydrocarbons in automobile exhaust becomes more difficult as emission control systems and engines become more efficient. Nevertheless, state and federal regulations that will take effect over the next few years mandate that more chemically specific information be reported on ten times less total emissions than the current standard. To address this need, fast and sensitive real-time on-line methods of analysis are actively under development.

One potential real-time on-line analytical method is based on atmospheric pressure corona discharge ionization (APCDI) and tandem mass spectrometry. Previous work [1] has shown the utility of this technique via charge exchange ionization under dry conditions. This report describes the use of nitric oxide (NO) as a selective reagent gas for charge exchange and charge attachment ionization under APCDI conditions. Nitric oxide is a well-characterized reagent gas that exhibits a variety of ionization mechanisms under classical chemical ionization (CI) conditions [2-5]. Nitric oxide can ionize by charge attachment $\left[\mathrm{M}+\mathrm{NO}^{+}\right]$, charge exchange $\left(\mathrm{M}^{+}\right)$, or hydride extraction $\left[\mathrm{M}-\mathrm{H}^{+}\right]$. The use of selective reagent gases in classical $\mathrm{CI}$ is a common

Address reprint requests to Dr. Mark A. Dearth, Ford Motor Co., Mail drop SRT. 3061, P.O. Box 2053, Dearborn, MI 48121-2053. practice; however, it is not a common practice with APCDI. Some early workers used selective reagent gases with APCDI including benzene [6] and nitric oxide [7] to enhance the ionization of hydrophobic compounds, such as PAH or PCB [8]. The utility of these ionization methods, however, could not be fully realized until tandem mass spectrometry became a common separation technique.

Fundamental studies of APCDI $[6,7,9]$ have shown that water is a significant potential interferent in ionization processes that rely on more energetic reagent gases (such as nitric oxide). In APCDl, charge exchange from more energetic charged species, to compounds with lower ionization potentials, occurs rapidly and with great efficiency. This is due to the large number of intermolecular collisions that occur at atmospheric pressure [6]. In fact, ionization efficiency can approach $100 \%$.

With samples in ambient air or vehicle exhaust, the natural abundance and low ionization potential of water make protonated water the predominant reagent ion in the APCDI source. In the past, this has limited the application of APCDI to compounds ionized by proton transfer reactions. Unfortunately, this restriction has limited APCDI to hydrophilic compounds and has excluded the measurement of most hydrocarbons. However, under dry conditions, nitric oxide-assisted 
APCDI can be used to ionize a wide variety of hydrocarbons with diverse functional groups $[7,9]$. Fundamental parameters that influence the nitric oxide ionization process are reported here, including nitric oxide concentration, water concentration in the ionization region, and ion source temperature.

Our goal was to determine a "robust" APCDI process suitable for the analysis of such diverse compounds as methanol and ethanol, formaldehyde and acetaldehyde, benzene, toluene, and other alkylbenzenes, methyl tert-butyl ether (MTBE), and the olefins including ethene and 1,3-butadiene, hereafter referred to collectively as hydrocarbons. A "robust" ionization process would be one unaffected by changes in the sample matrix composition, especially water. The species noted are important components of vehicle exhaust and are of regulatory or environmental interest. The remainder of this report will present the experimental conditions determined and the quantitation methods devised by using nitric oxide as an $\mathrm{APCDI}$ reagent gas and that result in a more robust APCDI process.

\section{Experimental}

Materials. Synthetic gas blends of hydrocarbons were commercially prepared (Scott Specialty Gases) and calibrated by gas chromatography-flame ionization detection analysis. Typically, hydrocarbon standard concentrations were about $1 \mathrm{ppm}$ in dry nitrogen, with as many as 24 compounds per gas cylinder. Gas standards were further diluted by using the boil-off from a liquid nitrogen dewar and $99.999 \%$ pure synthetic air (AIRCO Industrial Gases). Nitric oxide, at $9000 \mathrm{ppm}$ in nitrogen, was diluted with nitrogen or air to the required concentration.

The passage of air through a water-filled impinger produced known hydration levels of synthetic air. The impinger was maintained at room temperature, and saturation vapor pressure was determined from air temperature and barometric pressure. This hydrated air was then mixed with other dry gases, at predetermined ratios, to produce known water concentrations within the ionization region.

Methods. All gases were metered with mass flow controllers (Matheson, Inc., East Rutherford, NJ) and were transported and mixed in Teflon ${ }^{\text {or }}$ or stainless steel tubing. For calibration purposes, synthetic gas standards of hydrocarbons were serially diluted by using mass flow controllers with flow rates ranging from 10 $\mathrm{mL} / \mathrm{min}$ to $10 \mathrm{~L} / \mathrm{min}$. The mass flow controllers were sized so that the controlled flows were between 50 and $100 \%$ of full scale. Thus, the mass flow controllers were operated in the range of highest accuracy for the desired flow rate. The mass flow controllers were used as calibrated by the manufacturer. Typical gas flow rates through the ionization region were $5-7 \mathrm{~L} / \mathrm{min}$.
Mass spectrometer modifications. All mass spectrometric measurements were made with a Sciex Instruments (Thornhill, Ontario) API-III triple quadrupole mass spectrometer equipped with an atmospheric pressure corona discharge ionization (APCDI) source. The ionization region of this instrument is housed in a stainless steel chamber that is about $4.5 \mathrm{~L}$ in volume and has all gas infiltration points sealed with rubber gaskets or o-rings. The ionization chamber is additionally equipped (in-house) with a heating blanket and is heated to $100^{\circ} \mathrm{C}$.

Additional modifications to the gas inlet system also were made in-house. The schematic in Figure 1 depicts these changes. Briefly, the standard $2.2-\mathrm{cm}-\mathrm{o} . \mathrm{d}$. glass inlet tube is fitted with a concentric $0.63-\mathrm{cm}-0 . d$. glass tube afixed by a weld joint through the side of the 2.2-cm-o.d. tube, at an external point of the ion chamber. A copper block is machined to fit snugly over the concentric $0.63-\mathrm{cm}$ tube and snugly inside the $2.2-\mathrm{cm}-\mathrm{a}, \mathrm{d}$. inlet tube. This block is drilled to accept two $0.31-\mathrm{cm}$ cartridge heaters and a thermocouple for feedback control of heating. With this arrangement, gases passing through the concentric inner glass tube can be heated to above $350{ }^{\circ} \mathrm{C}$. A sleeve made of machinable ceramic (MACOR) is used to constrict the gases that exit the glass inlet to a narrow region that surrounds the corona discharge needle and the area in close proximity to the inlet orifice. The approximate volume of the ionization region within the ceramic sleeve is $3.5 \mathrm{~mL}$. At a flow rate of $7 \mathrm{~L} / \mathrm{min}$, this volume is swept more than 30 times per second.

Mass spectrometer measurements. Surveys of parent and daughter ions from nitric oxide ionization were conducted in the full scan mode, with typical scan rates of $1 \mathrm{~s}^{-1}$ across 15-200 u. For tandem mass spectrometry, argon was used as a collision gas at a density of $350-400 \times 10^{12}$ molecules $/ \mathrm{cm}^{2}$. Collision energy was 9-20 eV. Selected parent-daughter transitions were monitored for calibration and quantitation, with a dwell time of 50-100 ms per ion pair; see Table 1 for specific parent-daughter ion pairs that were monitored. Calibration and quantitation were accomplished by postrun processing with software supplied with the instrument.

\section{Results and Discussion}

\section{Prerequisite Conditions}

The concentration of water in the APCDI source must be very low (low parts per million) before nitric oxide can be used as a reagent gas. Under dry conditions, small amounts of nitric oxide will be formed by the corona discharge from the interaction of $\mathrm{N}_{2}^{+}$and $\mathrm{O}_{2}$ [9]. The appearance of a small $\mathrm{NO}^{+}$peak in the background spectrum of synthetic air is a good indicator of the degree of dryness of the ion source region. Once this state is achieved, the addition of small amounts of 


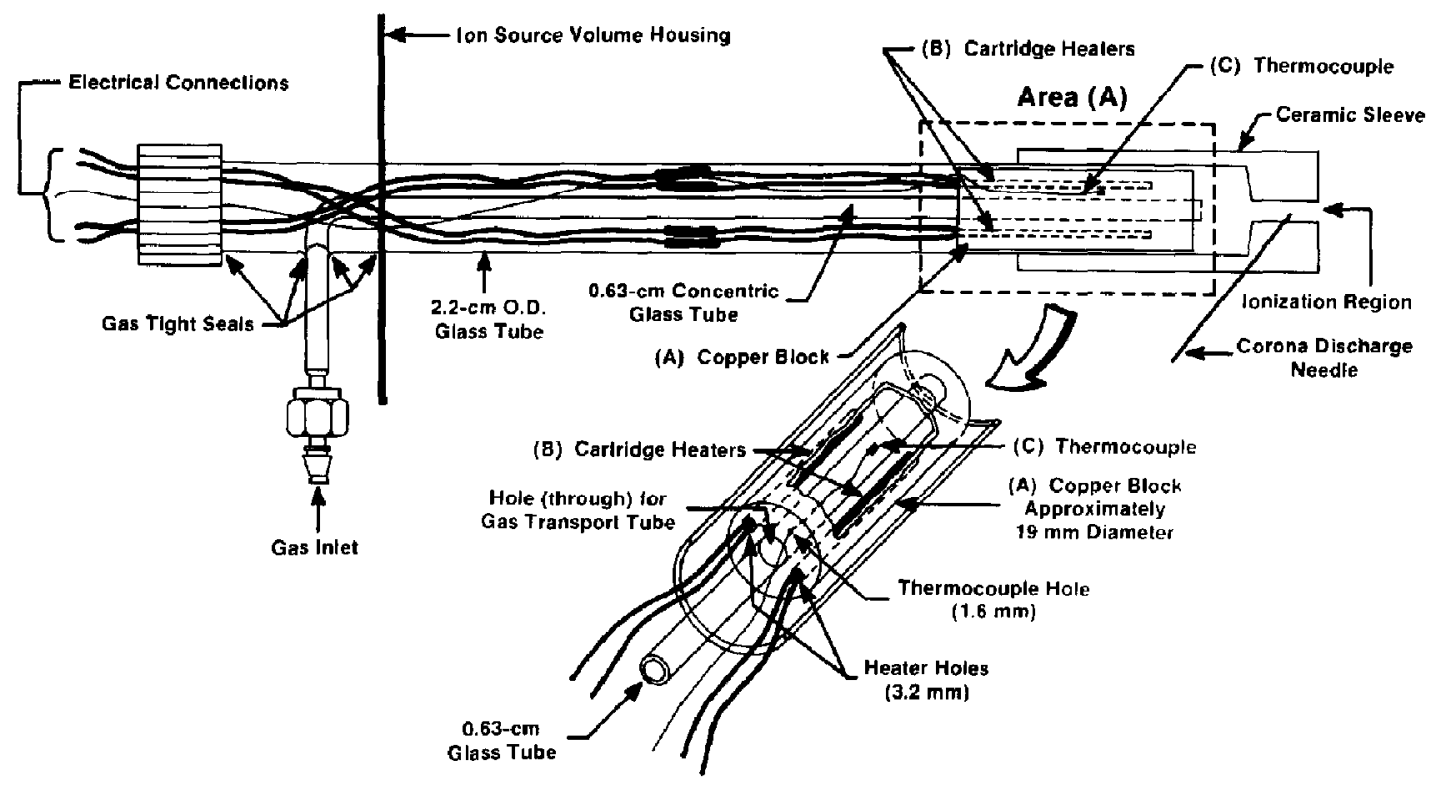

Area (A) Detail

Figure 1. Glass inlet (2.2-cm-0.d.) modifications for heating sample gases to $100-350^{\circ} \mathrm{C}$. A copper block $(A)$ is heated by two small cartridge heaters $(B)$ and temperature controlled by a thermocouple (C) and feedback circuit (not shown).

additional nitric oxide will bias the background spectrum so that $\mathrm{NO}^{+}$is the predominant ion and ions due to water will be attenuated [7]. These conditions can be achieved routinely by heating the lightly sealed ion source region and by using very dry gases as a continuous purge.

\section{Ionization Mechanisms}

Preliminary mass spectral investigations were conducted to determine the ionization mechanisms and the resulting ions for various hydrocarbons. Subsequent tandem mass spectra were acquired for the predominant molecular or pseudomolecular ions and their daughter ions. These tandem mass spectra, which contain the predominant pseudomolecular ion and coliision-induced fragment ions, are presented and discussed here. The results of these investigations will be discussed based on the functional group class for each type of compound.

Alkanes. Nitric oxide ionizes linear and branched alkanes by hydride abstraction and forms an $\left[\mathrm{M}-\mathrm{H}^{+}\right]$ pseudomolecular ion. This is the same ionization process observed for alkanes under low pressure nitric oxide CI conditions [10]. Full scan parent-daughter ion mass spectra were acquired for $n$-nonane, 2,2,4-trimethylpentane, 3-methylhexane, and methylcyclopentane and are shown in Figure 2. Daughter ion fragments have been labeled with tentative structures and are consistent with known alkyl fragmentation path- ways. For instance, $n$-nonane fragments by sequential loss of methylene units and 2,2,4-trimethylpentane fragments more preferentially to $m / z 57$, the tert-butyl cation.

Alkenes. The three potential ionization mechanisms (charge attachment, charge exchange, and hydride abstraction) are observed in the interaction of $\mathrm{NO}^{+}$with the alkenes. In Figure 3, five parent-daughter ion mass spectra are presented. We observed that trans-2-butene and 1,3-butadiene form $\left[\mathrm{M}+\mathrm{NO}^{+}\right]$charge attachment complexes. Collision-induced dissociation results in the loss of NO from the $\left[\mathrm{M}+\mathrm{NO}^{+}\right]$parent ion and then subsequent fragmentation consistent with the alkyl structure. In contrast, cis-2-pentene and 2-methylpropene produce $\mathrm{M}^{+}$charge exchange ions and the requisite alkyl fragment ions. Finally, 1-methylcyclopentene undergoes hydride abstraction to form the $\left[\mathrm{M}-\mathrm{H}^{\prime}\right]$ ion, which can be induced to fragment to smaller alkyl product ions. These processes previously have been observed for NO ionization of alkenes under low pressure CI conditions [11], with the different ionization processes also noted [3] for the cis- and trans-alkene isomers.

Alkylbenzenes. Alkylbenzenes are ionized by forming a nitric oxide charge attachment complex. Representative parent-daughter ion mass spectra for the alkylbenzenes are found in Figure 4. The $\left[\mathrm{M}+\mathrm{NO}^{+}\right]$parent ion can be induced to fragment to produce the $\mathrm{M}^{+}$ ion, which may undergo additional fragmentation. The 
Table 1. Ions monitored and relative sensitivities for specific compounds or compound classes ${ }^{\mathrm{a}}$

\begin{tabular}{|c|c|c|}
\hline Compounds & $P / D$ & icps/ppm $(\times 106)$ \\
\hline \multicolumn{3}{|l|}{ Alkanes } \\
\hline Branched octanes & $113 / 57$ & 0.76 \\
\hline Branched heptanes & $99 / 57$ & 0.53 \\
\hline Branched hexanes & $85 / 43$ & 0.48 \\
\hline Normal alkanes $\left(>C_{8}\right)$ & & 0.0008 \\
\hline \multicolumn{3}{|l|}{ Alkenes } \\
\hline 1,3-Butadiene & $84 / 54$ & 1.65 \\
\hline cis-2-Pentene & $100 / 69$ & 0.69 \\
\hline Pentenes & $100 / 70$ & 0.56 \\
\hline Propene & $72 / 30$ & 0.41 \\
\hline Butenes & $86 / 56$ & 1.53 \\
\hline Butenes & $56 / 41$ & 0.40 \\
\hline Ethene & $57 / 41$ & 0.03 \\
\hline Ethene & $56 / 29$ & 0.001 \\
\hline \multicolumn{3}{|l|}{ Aromatics } \\
\hline$C_{3}$ Benzenes & $150 / 120$ & 4.14 \\
\hline $\mathrm{C}_{3}$ Benzenes & $150 / 91$ & 0.04 \\
\hline $\mathrm{C}_{2}$ Benzenes & $136 / 106$ & 5.76 \\
\hline $\mathrm{C}_{2}$ Benzenes & $136 / 91$ & 0.30 \\
\hline Toluene & $122 / 92$ & 5.00 \\
\hline Benzene & $108 / 78$ & 3.43 \\
\hline \multicolumn{3}{|l|}{ Oxygenates } \\
\hline Formaldehyde & $60 / 30$ & 0.43 \\
\hline Methanol & $62 / 30$ & 0.37 \\
\hline Methyl tert-butyl ether & $118 / 57$ & 0.34 \\
\hline Methyl tert-butyl ether & $89 / 57$ & 0.32 \\
\hline Ethanol & $76 / 45$ & 0.19 \\
\hline Acetaldehyde & $74 / 43$ & 0.02 \\
\hline
\end{tabular}

${ }^{a} P / D$ is the parent ion and the subsequent daughter ian and icps is the ion counts per second (in millions) per compound in the ion source.

degree to which the $\mathrm{M}^{+}$ion fragments is roughly proportional to the size and number of alkyl substituents on the benzene ring; more $\left[\mathrm{M}-\mathrm{CI}_{3}^{+}\right]$is observed from the $C_{3}$ benzenes than the $C_{2}$ benzenes, and more from the $C_{2}$ benzenes than from toluene. Fragmentation of benzene and toluene $\mathrm{M}^{+}$ions is not observed. Benzene forms the strongest $\left[\mathrm{M}+\mathrm{NO}^{+}\right]$ adduct, but it can be induced to lose neutral NO by collision-induced dissociation, either in the collision cell (quadrupole 2) or in the region between the inlet orifice and the first quadrupole. Use of the orifice and quadrupole rod offset voltage to control the species ubserved will be discussed later (see the section entitled Orifice/rod offset voltage).

Oxygenates. The oxygenates studied here (see Figure 5) all exhibit charge attachment adducts as the predoninant product iun. The parent-daughter ion mass spectra depict cleavage of the $\left[\mathrm{M}+\mathrm{NO}^{+}\right]$adduct ion to yield either $\mathrm{M}^{+}$for MTBE and $\left[\mathrm{M}-\mathrm{H}^{+}\right]$for acetaldehyde or $\mathrm{NO}^{+}$for methanol and formaldehyde. These results are in contrast to previous work performed with low pressure nitric oxide $\mathrm{CI}$ [4], where $\left[\mathrm{M}-\mathrm{H}^{1}\right]$ for primary alcohols and aldehydes and $\left[\mathrm{M}-\mathrm{OCH}_{3}^{+}\right]$for MTBE were observed.
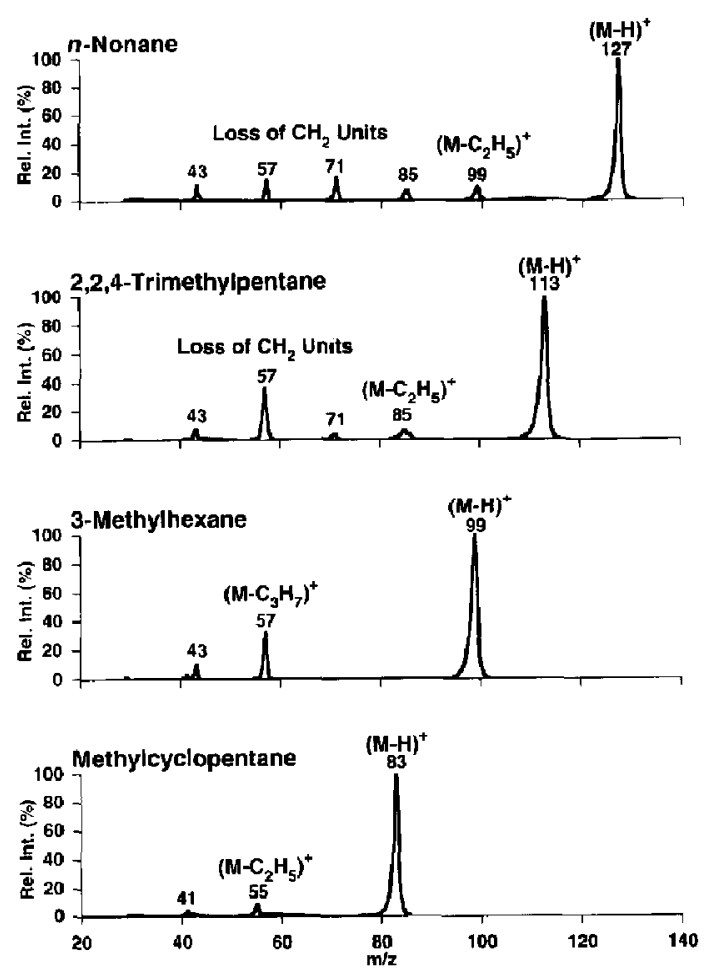

Figure 2. Parent-daughter ion mass spectra of selected alkanes. $n$-Nonane represents the linear alkanes, 3-methylhexane represents secondary carbon-containing alkanes, 2,2,4-trimethylpentane represents tertiary carbon-containing alkanes, and methylcyclopentane represents cyclic alkanes. In all cases, NO-assisted APCDI produces the [M- $\mathrm{H}^{\dagger}$ ] major (parent) ion, which was collisionally dissociated to produce the subsequent daughter ion fragments.

\section{Factors that Affect NO APCDI}

The most important parameter investigated for its impact on nitric oxide-assisted APCDI was the concentration of water in the sample gases. The importance of water is twofold: (1) it is an obvious interferent to APCDI when it is more than a trace component and (2) it makes up about 2 and $13 \%$ (by volume), respectively, of ambient air and vehicle exhaust. Other parameters that are important to nitric oxide-assisted APCDI include the concentration of nitric oxide, instrumental variables, and sample temperature. These topics are discussed in succeeding text.

Water concentration. At low parts per million concentrations water inlibits charge exchange ionization of hydrocarbons. A detailed understanding of this inhibitory effect was needed to affect methods of circumvention. Sludies were carried out with compounds whose ionization are most strongly inhibited-the alkylbenzenes.

Under very dry ion source conditions without nitric oxide addition, benzene and alkylbenzenes can be ion- 

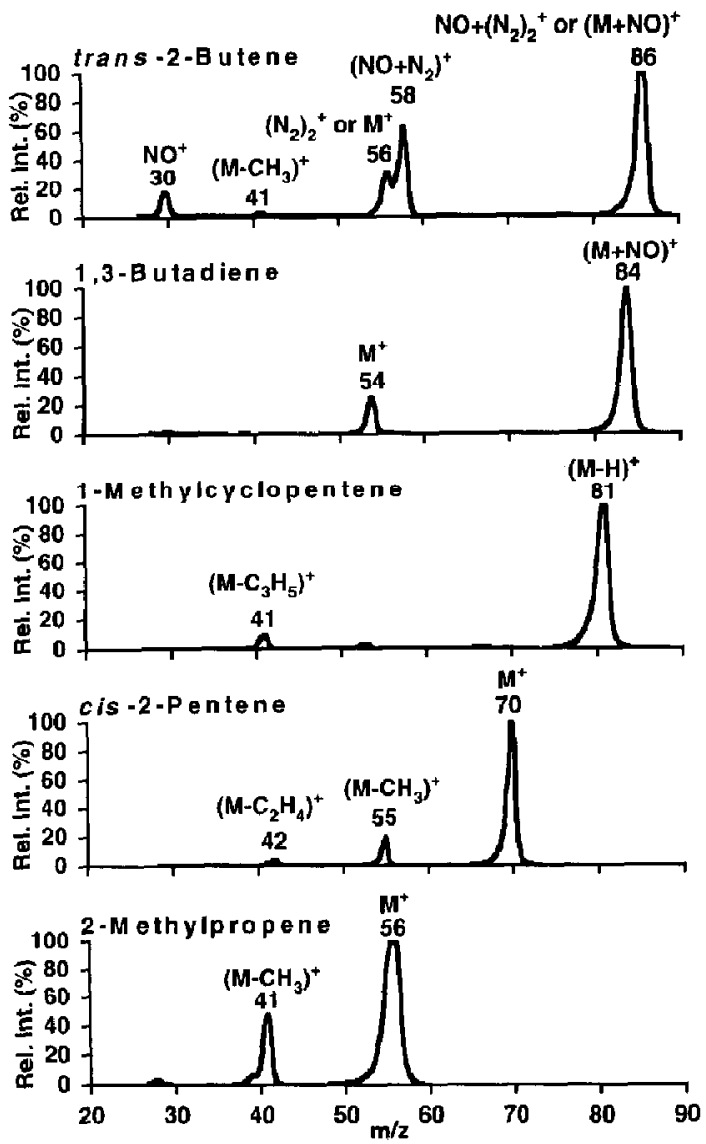

Figure 3. Parent-daughter ion mass spectra of selected alkenes. The alkenes shown were selected to represent various functionalities: cis-2-pentene and trans-2-butene represent the cis and trans conformations, 1,3-butadiene represents the dienes, 2-methylpropene represents an alkene with branching, and 1-methylcyclopentene represents cyclic alkenes.

ized by charge exchange from $\mathrm{N}_{2}^{+}, \mathrm{O}_{2}^{+}$, or the small amount of $\mathrm{NO}^{+}$formed in dry air [1]. However, as the concentration of water increases, charge exchange ionization is inhibited. Figure 6 contains plots of the average relative response of the alkylbenzenes at two temperatures, normalized to their response with no water versus added water vapor. Plot $A\left(320^{\circ} \mathrm{C}\right)$ will be discussed later. As shown by data plot B $\left(100^{\circ} \mathrm{C}\right)$, when the concentration of water reaches $100 \mathrm{ppm}$, the average signal of the aromatic compounds drops by more than $45 \%$. The expected range of water concentration in our target sample (diluted auto exhaust) is 0-100 ppir.

A possible method to mitigate the negative effect of water was suggested by Sunner et al. [12], who found that heating the gas stream improved the APCDI (by proton transfer from water) of marginally basic compounds. The reported improvements were attributed to the reduction in the size of the neutral solvent shell that forms around a protonated water ion. By logical
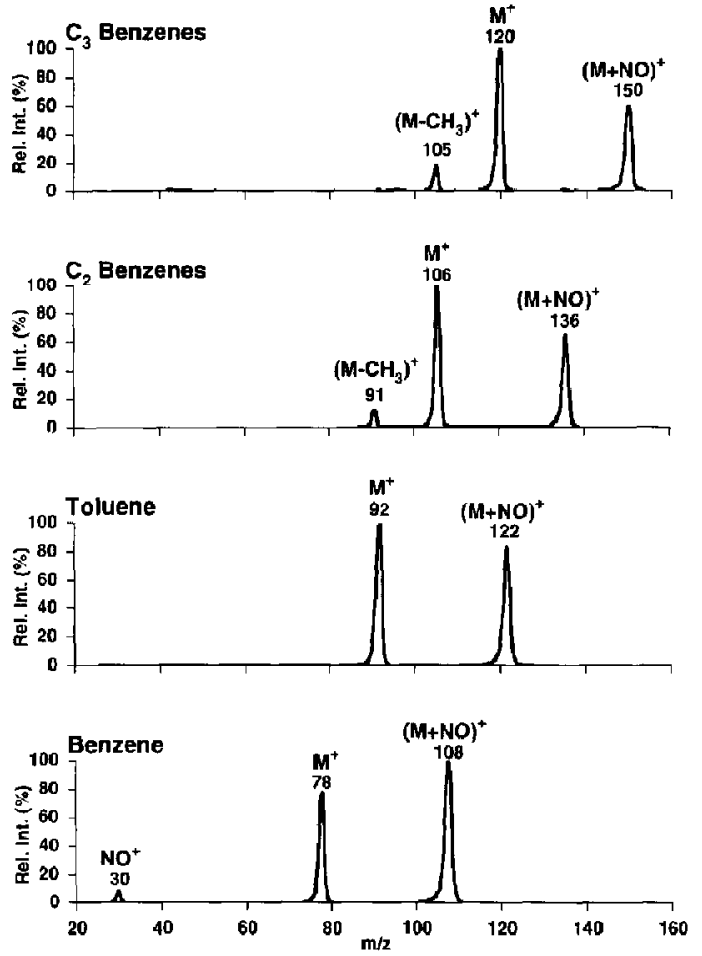

Figure 4. Parent-daughter ion mass spectra of selected alkyl benzenes. $C_{3}$ benzenes represent trimethylbenzenes and ethylmethylbenzenes; $C_{2}$ benzenes represent ethylbenzene and the xylenes.

extension, it is likely that $\mathrm{NO}^{+}$also is quickly solvated by neutral water molecules, which reduces the ability of $\mathrm{NO}^{+}$to interact with hydrophobic compounds. Thus a reduction of the neutral water solvent shell surrounding $\mathrm{NO}^{+}$should have a positive influence on nitric oxide ionization of hydrophobic compounds.

We observe that heating the sample gases does enhance charge exchange ionization, even under (nominally) dry conditions. Figure 7 is a plot of the ion signals of the alkylbenzenes normalized to the signal at $100{ }^{\circ} \mathrm{C}$ and plotted against sample gas temperature. Heating has a positive and dramatic impact on the ionization efficiency of the alkylbenzenes; a factor of 3-18 improvement was observed at $350^{\circ} \mathrm{C}$. This improvement is strongly dependent on the compound and the specific ion monitored. The ions monitored in Figure 7 are as follows: 120 represents the $\mathrm{M}^{+}$ion for the $\mathrm{C}_{3}$-substituted benzenes, 108 is the $\left[\mathrm{M}+\mathrm{NO}^{+}\right]$ adduct ion for benzene, 106 is the $\mathrm{M}^{+}$ion for $\mathrm{C}_{2}$-substituted benzenes, 92 is the $\mathrm{M}^{+}$ion for toluene, and 78 is the $\mathrm{M}^{+}$ion for benzene.

Returning to Figure 6, the effect of heating in the presence of added water is shown by plot $A$ for gases heated to $320^{\circ} \mathrm{C}$. The addition of water to the inlet gases still depresses the response of the alkylbenzenes, but by an average of only $15 \%$ with 100-ppm added water vapor. This is a factor of 3 (15 to $45 \%$ ) improve- 

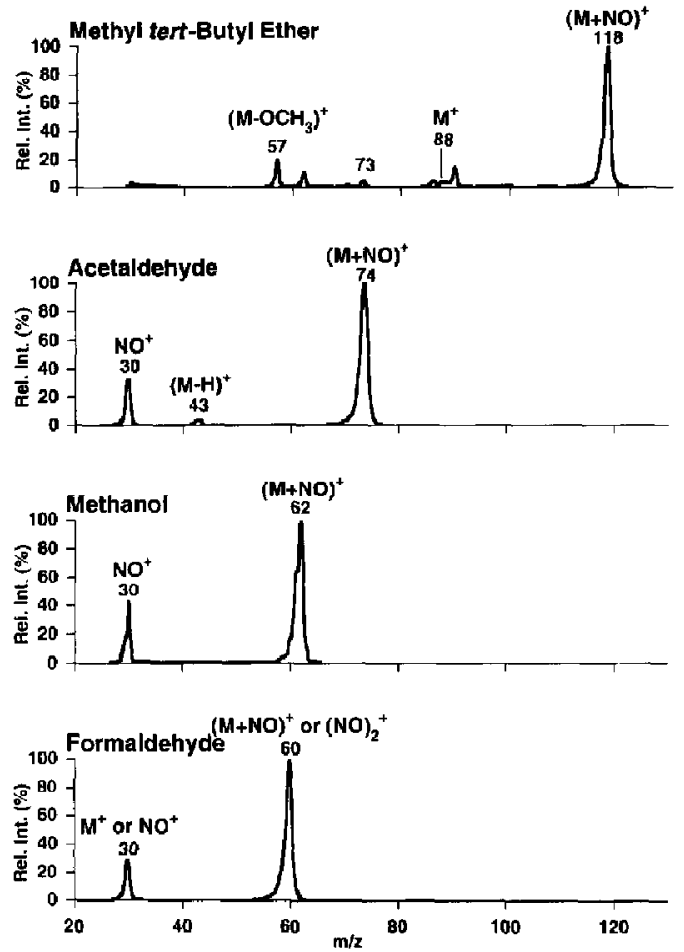

Figure 5. Parent-daughter ion mass spectra of several oxygenated hydrocarbons. Methyl tert-butyl ether represents the ethers, acetaldehyde and formaldehyde represent the aldehydes, and methanol represents the alcohols.

ment in the robustness of APCDI when compared to the loss of signal due to 100 -ppm water at $100{ }^{\circ} \mathrm{C}$ plot B.

Nitric oxide concentration. The improvements due to heating include additional sensitivity and a more robust ionization process, but the addition of nitric oxide

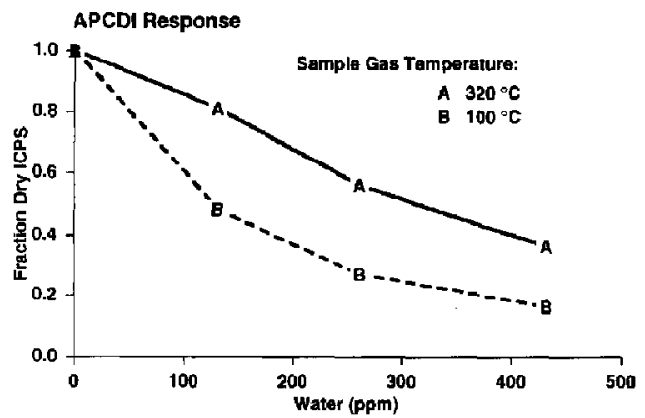

Figure 6. The average response of the alkybenzenes to nitric oxide-assisted APCDI upon addition of water to the sample gases. The response is nomalized to the anhydrous response which is equal to 1 . Plot $A$ represents the relative response with the sample gases heated to $320^{\circ} \mathrm{C}$. Plot $B$ represents the relative response of unheated (nominally $100{ }^{\circ} \mathrm{C}$ ) sample gases and increasing humidity.

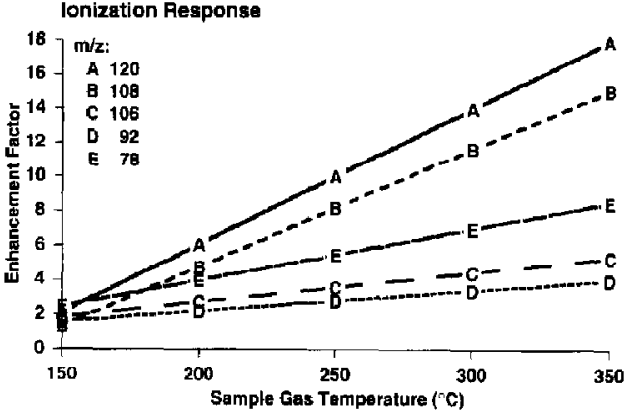

Figure 7. Ionization enhancement plotted as a function of sample gas temperature. The ionization response was set equal to 1 at $100{ }^{\circ} \mathrm{C}$. The traces are labeled as follows: 120 is trimethylbenzene, 108 is benzene, 106 is xylene, 92 is toluene, and 78 is benzene. The numbers $(120,108,106,92$, and 78$)$ correspond to the mass of either the $\mathrm{M}^{+}$or $\mathrm{M}+\mathrm{NO}^{+}$monitored.

to a dry ion source also improves ionization efficiency. The plot in Figure 8 shows an average eight-fold improvement in charge exchange ionization efficiency for the alkylbenzenes at 2000-3000-ppm nitric oxide concentrations. The slope of the ionization response curve begins to flatten out at 2000-ppm nitric oxide. Therefore, 3000-ppm (or more) nitric oxide was used for subsequent experiments.

Significant improvements in measurement capability result from the combination of the addition of nitric oxide and heating the sample gases. The overall improvement in sensitivity is a factor of between 20-100 (3-18 due to heating and 8 due to nitric oxide) relative to that obtained at $100{ }^{\circ} \mathrm{C}$ without nitric oxide. The ionization process is also a factor of 3 times more robust in the presence of added water (45 versus $15 \%$ loss of signal at 100-ppm water).

Curtain gas flow rate. A counterflow of pure dry nitrogen is used to decluster and exclude nonionized atmospheric gases from the high vacuum region of the Sciex instrument [6]. However, a significant interference ion

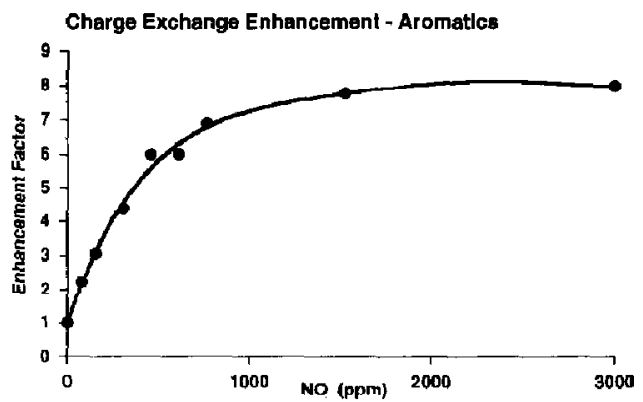

Figure 8. Relative response (icps set $=1$ with NO concentration $=0$ ) plotted as a function of the nitric oxide concentration. Data were obtained at an ion source temperature of $100{ }^{\circ} \mathrm{C}$. The response curve represents the average of the responses obtained for benzene, toluene, a xylene, and a trimethylbenzene. 


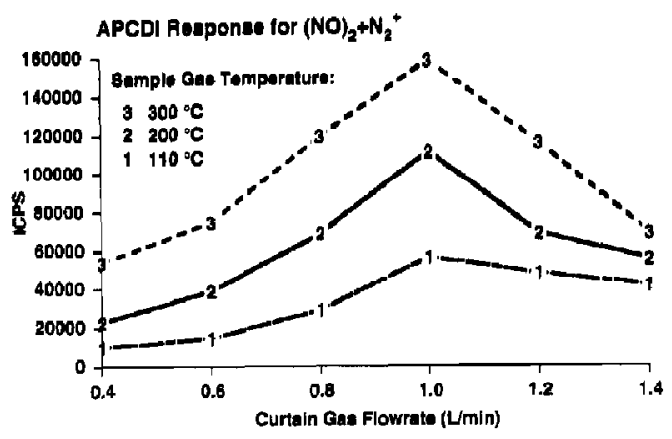

Figure 9. Ion intensity (icps) of $\left(\mathrm{NO}_{2} \mathrm{~N}_{2}^{+}\right.$as a function of curtain gas flow rate at three different temperatures

results from (NO) ${ }_{2} \mathrm{~N}_{2}^{+}$at $m / z 88$ that is produced by clustering of $\mathrm{NO}^{+}$with the neutral $\mathrm{N}_{2}$ curtain gas. This ion is the same mass as one potential ion of MTBE $\left(\mathrm{M}^{+}\right)$. The abundance of the interference ion was observed to be related to the flow of curtain gas; these data are plotted in Figure 9. To minimize the formation of this interfering molecule, the curtain gas flow rate was lowered from 1.2 to $0.6 \mathrm{~L} / \mathrm{min}$. The lower curtain gas flow rate also improved overall ion transmission and, therefore, sensitivity.

Orifice/rod offset voltage. The ratio of orifice (OR) to (quadrupole) rod offset ( $\mathrm{RO}$ ) voltage can range from 12 or more. In practical terms, the orifice and rod offset voltage values are generally $32-70$ and around $30 \mathrm{~V}$, respectively. At higher orifice voltages, the large polential $(30-40 \mathrm{~V})$ between the otifice and the first quadrupole accelerates the ions in this region. This region has a fairly high neutral gas density, which means energetic collisions between neutrals and fast moving ions can be induced [13]. This region can be used to dissociate solvent-ion clusters or to fragment molecules, depending on the energies involved. The effect of increasing the orifice voltage (with a fixed rod offset voltage of $30 \mathrm{~V}$ ) is shown in Figure 10 . The ratios of $\mathrm{M}^{+}$to $\left[\mathrm{M}+\mathrm{NO}^{+}\right]$adduct ion are plotted for several alkylbenzenes. At higher orifice voltages, nitric oxide is efficiently stripped from the alkylbenzene ion. It can also be deduced from the slope of the data that the benzene-nitric oxide adduct is the most strongly

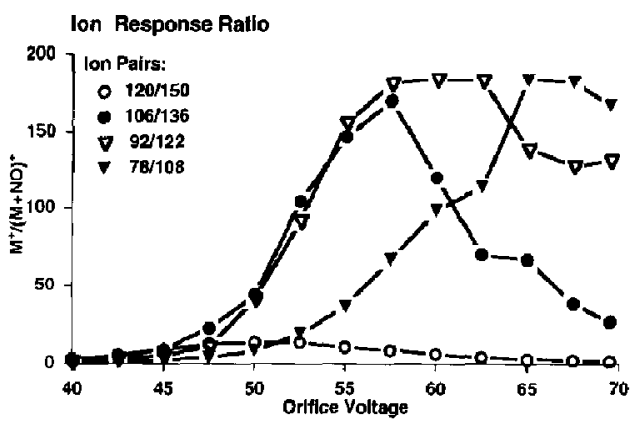

Figure 10. The effect on the ratio of $\mathrm{M}^{+} / \mathrm{M}+\mathrm{NO}^{+}$of increasing orifice voltage (OR) with the quadrupole offset voltage (RO) held constant (i.e., $30 \mathrm{~V}$ ). The $\mathrm{M}^{+} / \mathrm{M}+\mathrm{NO}^{+}$traces are labeled as follows; $120 / 150$ is trimethylbenzene, $106 / 136$ is xylene, $92 / 122$ is toluene, and $78 / 108$ is benzene. The numbers correspond to the masses (mass-to-charge ratio) monitored.

bound of these adducts. The practical implication of this is that two equally representative ions can be selected for monitoring the same compound simply by selecting the proper $\mathrm{OR} / \mathrm{RO}$ voltage ratio.

To summarize our findings, if water is reduced to less than $100 \mathrm{ppm}$ in the ion source (by simple dilution of the sample with dry air or nitrogen), if nitric oxide is added to $3000 \mathrm{ppm}$ in the ion source, and if the sample gases are heated to $300^{\circ} \mathrm{C}$, then the impact of the most significant matrix interferent (water) can be reduced to a range of $\pm 7 \%$, with sufficient sensitivity to measure most compounds of interest in ambient air. This satisfies our definition of a "robust" APCDI ion source.

\section{Quantitation}

Compound list and tandem mass spectrometry quantitation. A list of the most abundant or photochemically reactive compounds of interest to the automotive emissions measurement community is found in Table 2 .

Based on the mass spectra described in the section Ionization Mechanisms, parent ion-daughter ion pairs were selected for tandem mass spectrometry quantitation. The final quantitation list is found in Table 1 . The first column of Table 1 contains the compound class or name; column two ( $P / D)$ lists the specific parent-

Table 2. List of taret chemical species in vehicle emissions

\begin{tabular}{llll}
\hline Methane & Ethene & Formaldehyde & Methanol \\
Propene & Acetaldehyde & Ethanol & 1,3-Butadiene \\
1-Butene & 2-Methylpropene & cis-2-Butene & trans-2-Butene \\
n-Butane & 2-Methyl-1,3-butadiene & 2-Methyl-2-butene & cis-2-Pentene \\
trans-2-Pentene & 2-Methylbutane & Benzene & 2-Methylpentane \\
3-Methylpentane & Methyl tert-butyl ether & Toluene & 2,3-Dimethylpentane \\
Ethylbenzene & $o$-Xylene & $m$-Xylene & $p$-Xylene
\end{tabular}


daughter ion pair monitored, and column three (ICPS $/ \mathrm{ppm}$ ) lists the relative response of each compound. Note that tandem mass spectrometry is unable to distinguish between many configurational isomers. For instance, the $\mathrm{C}_{3}$ - and $\mathrm{C}_{2}$-substituted benzenes are each quantified with one parent-daughter ion pair. The responses of the the various isobaric alkylbenzenes were assumed to be the same based on determination of individual response factors for representative compounds from each isobaric group.

Sensitivities. A value of ion counts per second per part per million compound (icps/ppm) is found in the last column of Table 1. These values are not the absolute sensitivity for a given compound, but should be used as a relative comparison of the sensitivity of this method for different compounds within the list. To determine absolute sensitivity, one may use the following response for benzene: $3 \mathrm{ppb}$ of benzene produces about $1 \times 10^{6}$ icps. The standard deviation of the background signal is about 1000 icps. Thus a calculated limit of quantitation for benzene $(S / N=5)$ is 5000 icps, or about $0.015 \mathrm{ppb}$. The practical detection limit, given the need to maintain water concentration in the ion source at or below $100 \mathrm{ppm}$, would be a factor of 200 higher, or about $3 \mathrm{ppb}$ in air containing $100 \%$ relative humidity. This is sufficiently sensitive to measure benzene in urban ambient air. Sensitivities for other compounds can be estimated from these absolute values for benzene and their ratio of sensitivities in Table 1.

Relative sensitivities change with variations in the composition of the sample matrix. This effect is most pronounced with the aldehydes and alcohols. Water strongly inhibits the nitric oxide ionization of formaldehyde and to a lesser extent, acetaldehyde and the alcohols (data not shown). However, acetaldehyde and the alcohols readily form protonated ions $\left[\mathrm{M}+\mathrm{H}^{+}\right]$ and protonated water ion clusters $\left[\mathrm{M}+\mathrm{H}_{3} \mathrm{O}^{+}\right]$, with even small amounts of water (10-100 ppm) present, which means that they still might be quantified via protonation, whereas the alkenes and alkylbenzenes are quantified via nitric oxide ionization. Investigations are under way to determine optimum source conditions for simultaneous detection of all these compounds.

The two molecules that are most difficult to quantify are ethylene and formaldehyde. These two compounds present separate challenges to quantitation. Ethylene does not respond well to nitric oxide ionization (see Table 1). Furthermore, ethylene does not give easily characterized molecular or pseudomolecular ions, but instead forms small amounts of $\mathrm{M}^{+}$, $\left[2 \mathrm{M}+\mathrm{H}^{+}\right]$, and $\left[2 \mathrm{M}-\mathrm{H}^{+}\right]$ions. Further study is needed to determine if any of these ions can be used for quantitation.

Nitric oxide ionization of formaldehyde, as previously mentioned, is sharply attenuated by even traces of water. Protonated complexes of formaldehyde (the major alternative to NO ionization) do not provide sufficient sensitivity for quantitation at water concentrations that are tolerable for the other target compounds. Another possibility is to derivatize formaldehyde (and the other aldehydes) in the gas phase just prior to ionization [14]. The derivative would have to be volatile, selective, and easily ionized by nitric oxide or protonation.

These methods extend APCDI to a wider range of compounds than previously amenable to this type of ionization and reduce the sensitivity of APCDI to matrix interferences, especially water. The methods described herein reduce the effect of water (the most significant inferferent expected in ambient air or auto exhaust) on compound response factors to $\pm 7 \%$ (at worst), yet still provide sufficient sensitivity to quantify a wide variety of hydrocarbons at single parts per billion levels in real-world samples. These methods also are suitable for other applications such as interfacing gas chromatography to APCDI/mass spectrometry. Due to the very dry nature of the gas chromatography effluent and the need for highly sensitive and selective delection, nilric oxide-assisled APCDI would be eminently suited for this application.

\section{Acknowledgments}

This work was conducted under the auspices of the Environmental Research Consortium (ERC) and is part of Ford's contribution to the Auto Industry/Government Environmental Research (AIGER) CRADA. We thank our colleagues at Ford Research Labs, the ERC. and AIGER for their support and interest. We also thank I. Dzidic at Shell Oil for a helpful conversation.

\section{References}

1. Dearth, M. A.; Gierczak, C. A.; Siegl, W. O. S. Environ. Sci. Tectnol. 1992, 26, 1573-1580.

2. Polley, C. W.; Munson, B. Aral, Chem. 1983, 55, 754-757.

3. Hunt, D. F.; Harvey, T. M. Anal. Chem. 1975, 47, 2136-2141.

4. Chai, R.; Harrison, A. G. Anal. Chem. 1983, 55, 969-971.

5. Dzidic, I.; Petersen, H. A.; Wadsworth, P. A.; Hart, H. V. Anal. Chem. 1992, 64, 2227-2232.

6. Lane, D. A.; Sakuma, T.; Quan, E. S. K. In Poiynuclear Ammatic Hydrocatbons: Fourth International Symposium on Analysis, Chemistry, and Biology; Batelle Press; Columbus, $\mathrm{OH}$, 1980, pp 199-214.

7. Dzidic, L.; Carroll, D. D.; Stillwell, R. N.; Horning, E. C. Anat. Chem. 1976, 48, 1763-1768.

8. French, J. B.; Thomson, B. A.; Davidson, W. R.; Reid, N. M.; Buckley, J. A. In Mass Spectrometry in Environmental Sciences; Karasek, F. W.; Hutzinger, O.; Safe, S., Eds.; Plenum: New York, 1984, pp 101-121.

9. Siegel, M. W.; Fite, W. L. J. Phys. Chem. 1976, 80, 2871-2881.

10. Hunt, D. F.; Harvey, T. M. Anal. Chem. 1975, 47, 1965-1969.

11. Budzikiewicz, H.; Busker, E.; Brauner, A. Adv. Mass Spectrom. 1980, 84, 713-722.

12. Sunner, J.; Ikonomou, M. G.; Kebarle, P. Anal. Chem. 1988, 60. 1308-1313.

13. Kambara, H.; Kanomata, I. Mass Spectrosc. 1976, 24, 229-236 and 271-282.

14. Hughes, R.; March, R. Presented at the 4lst Annual ASMS meeting in San Francisco, CA, May 29-June 4, 1993. 